\title{
Attitudes to chemotherapy: comparing views of patients with cancer with those of doctors, nurses, and general public
}

\author{
Maurice L Slevin, Linda Stubbs, Hilary J Plant, Peter Wilson, Walter M Gregory, P Joanne Armes, \\ Susan M Downer
}

Abstract
Objective-To compare responses of patients with cancer with those of a matched control group, cancer specialists, general practitioners, and cancer nurses in assessing personal cost-benefit of chemotherapy.

Design-Prospective study of consecutively recruited patients with cancer and other groups by questionnaire; half of the patients received the questionnaire again three months after starting treatment.

Setting-A medical oncology ward of a London teaching hospital.

Subjects - 106 Patients with newly diagnosed solid tumours referred to the unit for consideration of treatment with cytotoxic chemotherapy, 100 of whom were able to complete the questionnaire. 100 Matched controls, 315 cancer doctors (238 radiotherapists and 77 medical oncologists), 1500 randomly chosen general practitioners, and 1000 randomly chosen cancer nurses.

Main outcome measures-Percentage chance of cure, prolonging life, or palliation of symptoms required to make treatment worth while with two hypothetical chemotherapy treatments, with severe and mild side effects respectively.

Results-Respondents to the questionnaire comprised 100 patients, 100 controls, $60(78 \%)$ medical oncologists, $88(37 \%)$ radiotherapists, 790 $(53 \%)$ general practitioners, and $303(30 \%)$ cancer nurses. Most patients were willing to accept intensive chemotherapy for a very small chance of benefit. The median benefit required to make the hypothetical intensive treatments worth while for patients compared with controls were: for chance of a cure (range 1 to $100 \%$ ) $1 \% v 50 \%$, for prolonging life (range three months to five years) 12 months $v$ 24-60 months, and for relief of symptoms (range 1 to $100 \%$ ) $10 \% v 75 \%$ respectively. There were no significant differences in the responses of the $\mathbf{5 0}$ patients completing the questionnaire on a second occasion. Doctors and nurses were less likely to accept radical treatment for minimal benefit compared with the patients (median scores $10-50 \%, 12-24$ months, and $50-75 \%$, for chance of cure, prolonging life, and relief of symptoms respectively). Significantly more patients than controls accepted treatments giving the minimal benefit for each category (cure 53.1 $v$ $19.0 \%, 67.0 v 35.0 \%$; prolonging life $42.1 v 10.0 \%$, $53.0 v 25.0 \%$; relief of symptoms $42.6 v 10.0 \%, 58.7 v$ $19.0 \%$ for intensive and mild treatments respectively, $\mathbf{p}<\mathbf{0 . 0 0 1}$ ) as was the case for comparison of patients with other groups.

Conclusion-Patients with cancer are much more likely to opt for radical treatment with minimal chance of benefit than people who do not have cancer, including medical and nursing professionals. This should be taken into account when discussing treatment options with patients and their relatives.

Correspondence to: Dr M L Slevin,

St Bartholomew's Hospital,

London EC1 7BE.

BrMed f 1990;300:1458-60
Until fairly recently the general public expected, and indeed was happy, to leave medical decision making to doctors. An increasing interest in consumer affairs has resulted in an awareness that personal responsibility for health care and participation in medical decision making are desirable and possible. Several North American studies have examined participation preferences. Cassileth et al sought the preferences of 256 patients with cancer.' ${ }^{1}$ Most patients in each age group preferred active participation, although, generally, younger patients wanted to be more involved. Degner and Russell asked 60 patients with cancer whether they preferred to "keep, share, or give away" control over decision making. ${ }^{2}$ Most preferred the option of shared control, usually with the doctor rather than a relative.

Increased participation in decision making means that patients need more information about their disease and treatment options. This has caused concern among health care workers, who thought that this might be depressing for patients. ${ }^{*}$ Cassileth et al found that patients were able to maintain hope, and in fact were generally more hopeful, despite having this information. ' Faden et al showed that doctors' and patients' opinions differed about the outcome of receiving information about drug treatment for epilepsy. ${ }^{+}$ Doctors believed that patients would have less confidence in their drugs if they were given more information whereas patients thought that they would be more likely to comply with treatments and would have more confidence in the prescribed drugs and in their doctors if given more information.

Two studies were conducted by McNeil et al in 1978 and $1981 .^{56}$ The first studied patients with stage $I$ or stage II cancer of the lung who had already received surgery or radiotherapy. The patients were interviewed individually about their attitudes to various periods of survival in hypothetical situations. Of the 14 patients with cancer who had participated, 12 were "risk averse" and considered the small chance of early death during surgery unacceptable. They preferred radiotherapy despite its lower five year survival rate. The second study examined the attitudes of healthy volunteers to quality of life rather than its quantity. Volunteers had to imagine that they had T3 carcinoma of the larynx and were asked whether they would prefer surgery, with its then current higher survival rate but loss of normal speech, or radiotherapy, with its lower survival rate but preservation of normal or nearly normal speech. In this hypothetical situation a fifth chose radiotherapy.

These North American studies showed that patients' and doctors' attitudes to treatment options of ten differ and that patients may welcome the opportunity to be included in the decision making process.

Our study examined the treatment preferences of patients with cancer in a British hospital by questionnaire and compared them with those of a matched control group, cancer specialists, general practitioners, and cancer nurses.

Subjects and Methods

QUESTIONNAIRE

We used a questionnaire describing two hypothetical treatments with chemotherapy: one representing a 
typical intensive chemotherapy regimen and the other a much milder chemotherapy regimen. The intensive regimen was said to have a considerable number of side effects and drawbacks, such as severe nausea and vomiting, hair loss, frequent use of needles and drips, frequent tiredness and weakness, admission to hospital for three or four days a month, decreased sexual interest (for the period of treatment), and possible infertility. The mild regimen was said to have fewer side effects and drawbacks, with only slight nausea and vomiting, no loss of hair, occasional use of needles and drips, some tiredness and weakness, and admission to hospital about once a month.

Subjects were asked to assess their willingness to have these two treatments in three possible circumstances; the first when the treatment offered a chance of cure, the second when cure was not possible but the treatment offered the chance of prolonging life, and the third when treatment was given only to relieve symptoms.

Patients were asked to rate the possible benefit that would make acceptance of these treatments worth while. Thus for the curative treatment they rated what chance of cure between $1 \%$ and $100 \%$ would make the treatment worth while. For the treatment designed to prolong life they were asked what duration, ranging from three months to five years, would make this treatment worth while, and when the treatment was given to relieve symptoms they were asked what chance of relieving symptoms, rated from $1 \%$ to $100 \%$, would make this treatment worth while. In all circumstances there was an option for saying that the treatment was unacceptable under any circumstances.

\section{SUBJECTS}

Patients-One hundred and six consecutive patients with solid tumours who were about to receive chemotherapy were asked to complete the questionnaire. Six patients were unable to complete it, three being too distressed and three being unable to understand the questions. Of the 100 patients remaining, 41 had small cell carcinoma of the lung and 59 had other types of tumour including ovarian carcinoma, cervical carcinoma, myeloma, and lymphoma. The median age of the patients was 60 (range 23 to 80 ), and there was no significant difference in age between men and women. The questionnaire was given to patients by a research nurse, who made it clear that she had no part in decisions about their treatment. It was given a second time to half of the patients chosen randomly after they had completed three cycles of treatment. Two patients who were approached thought that they could not repeat the questionnaire because their treatment with chemotherapy had failed and they were too distressed to consider a hypothetical situation.

Controls-One hundred controls matched for age, sex, ethnic origin, and occupation were obtained from the community. None of them or close members of their families had cancer or had previously had cancer. Patients with other diseases and hospital staff who had

TABLE I-Median scores (representing minimal benefit to make two hypothetical treatments acceptable in TABLE I-Median scores (representing minimal benefit to make two hypothetical treatments acceptable in
three circumstances) for controls, cancer nurses, general practitioners, cancer doctors, and patients with cancer

\begin{tabular}{|c|c|c|c|c|c|}
\hline & \multicolumn{5}{|c|}{ Subject group } \\
\hline & $\begin{array}{l}\text { Controls } \\
(n=100)\end{array}$ & $\begin{array}{l}\text { Cancer nurses } \\
\quad(n=303)\end{array}$ & $\begin{array}{l}\text { General practitioners } \\
\qquad(\mathbf{n}=790)\end{array}$ & $\begin{array}{l}\text { Cancer doctors } \\
\quad(n=148)\end{array}$ & $\begin{array}{l}\text { Patients } \\
(\mathbf{n}=100)\end{array}$ \\
\hline \multicolumn{6}{|l|}{ Intensive treatment: } \\
\hline Chance of cure (\%) & 50 & 50 & 25 & 10 & 1 \\
\hline Prolonging life (months) & $24-60$ & 24 & 24 & 12 & 12 \\
\hline Relief of symptoms (\%) & 75 & 50 & 75 & 50 & 10 \\
\hline \multicolumn{6}{|l|}{ Mild treatment: } \\
\hline Chance of cure $(\%)$ & 25 & 10 & 10 & 10 & 1 \\
\hline Prolonging life (months) & 18 & 12 & 12 & 6 & 3 \\
\hline Relief of symptoms (\%) & 50 & 25 & 25 & 25 & 1 \\
\hline
\end{tabular}

direct contact with patients were also excluded from the control group.

Cancer doctors-The questionnaire was sent to 238 radiotherapists and 77 medical oncologists, together with a covering letter requesting them to answer the questions as if they themselves had cancer. Responses were received from sixty medical oncologists $(78 \%)$ and 88 radiotherapists $(37 \%)$. The medical oncologists and radiotherapists circulated represented as complete a list of consultants and senior registrars in these specialties as could be obtained.

General practitioners-A direct postal company circulated the questionnaire to 1500 randomly chosen general practitioners. The company advised us that this would probably produce 100 responses, but in fact $790(53 \%)$ general practitioners responded.

Cancer nurses-Questionnaires were circulated randomly to 1000 of the 3500 members of the Royal College of Nursing Oncology Nursing Society, and 303 $(30 \%)$ responded.

Differences in proportions of subjects opting for each preference among the different groups were tested for significance using the $\chi^{2}$ test. Median scores were produced for each group as a summary statistic.

\section{Results}

Table I shows the median scores for each group for the intensive and mild treatments. The scores represented the minimal benefit that would make treatment acceptable to the subjects, the range for all groups being from the minimum acceptable to unacceptable.

Substantial differences were shown between the patients who had cancer and every other group. Most importantly, the patients with cancer gave very different results from the matched control group for both treatments in all circumstances. In general, the patients were most likely to accept intensive treatments for a potentially small benefit whereas the control group were least likely to accept intensive treatment and wanted the most benefit for any particular risk. After completing three months of treatment the responses of the 50 patients who completed the questionnaire a second time changed negligibly. No significant difference was shown when the first and second responses were compared for the intensive and the mild treatments, whether or not the patient had actually received an intensive or a milder treatment.

The responses of the medical oncologists, general practitioners, and cancer nurses fell between those of the patients and controls, with medical oncologists being more likely to accept radical treatments than general practitioners, who in turn were more likely to accept them than cancer nurses. Radiotherapists were the least likely of any group to accept treatment.

Table II shows the percentage of subjects in each group who would accept the intensive and mild treatments given minimal benefit for each category for cure, prolonging life, or relief of symptoms. It illustrates the dramatic difference between the patients and all other groups. Significantly more patients accepted treatments with much less benefit than any other group $(p<0.001)$, and the radiotherapists required more benefit from the treatments than any other group $(\mathrm{p}<0 \cdot 01)$.

\section{Discussion}

Presenting patients with treatment options is a complex and difficult process. On the one hand patients need sufficient information to allow them to make an informed decision, but on the other hand the doctor does not want to remove all hope and demoralise the patient. Thus most patients treated in a centre that specialises in the treatment of cancer are given infor- 
TABLE II-Respondents accepting intensive and mild treatments with a supposed minimum chance of effectiveness by subject group

\section{Subject group}

General

Controls Cancer nurses practitioners Radiotherapists Oncologists Patients

\begin{tabular}{|c|c|c|c|c|c|c|}
\hline \multirow[b]{2}{*}{ Intensive treatment: } & \multicolumn{3}{|c|}{ Cure $(1 \%)$} & & \multirow[b]{3}{*}{60} & \\
\hline & & & & & & \\
\hline Total respondents & 100 & 300 & 790 & 88 & & 96 \\
\hline $\begin{array}{l}\text { No (\%) of respondents accepting } \\
\text { treatment }\end{array}$ & 19 & $41(13 \cdot 5)$ & $98(12 \cdot 4)$ & $4(4 \cdot 5)$ & $12(20 \cdot 0)$ & $51(53 \cdot 1)$ \\
\hline \multicolumn{7}{|l|}{ Mild treatment: } \\
\hline Total respondents & 100 & 302 & 788 & 88 & 60 & 94 \\
\hline $\begin{array}{l}\text { No (\%) of respondents accepting } \\
\text { treatment }\end{array}$ & 35 & $118(38 \cdot 9)$ & $349(44 \cdot 3)$ & $24(27 \cdot 3)$ & $31(51 \cdot 7)$ & $63(67 \cdot 0)$ \\
\hline \multicolumn{7}{|c|}{ Prolonging life (by three months) } \\
\hline Intensive treatment: & & & & & & \\
\hline Total respondents & 100 & 302 & 790 & 86 & 60 & 95 \\
\hline $\begin{array}{l}\text { No }(\%) \text { of respondents accepting } \\
\text { treatment }\end{array}$ & 10 & $18(6 \cdot 0)$ & $26(3 \cdot 3)$ & 0 & $6(10 \cdot 2)$ & $40(42 \cdot 1)$ \\
\hline \multicolumn{7}{|l|}{ Mild treatment: } \\
\hline Total respondents & 100 & 303 & & 87 & 60 & \\
\hline $\begin{array}{l}\text { No }(\%) \text { of respondents accepting } \\
\text { treatment }\end{array}$ & 25 & $77(25 \cdot 4)$ & $215(27 \cdot 3)$ & $11(12 \cdot 6)$ & $27(45 \cdot 0)$ & $50(53 \cdot 0)$ \\
\hline \multirow{2}{*}{\multicolumn{7}{|c|}{ Relief of symptoms $(1 \%)$}} \\
\hline & & & & & & \\
\hline Total respondents & 100 & 303 & 790 & 87 & 60 & \\
\hline $\begin{array}{l}\text { No (\%) of respondents accepting } \\
\text { treatment }\end{array}$ & $10(10)$ & $18(5 \cdot 9)$ & $19(2 \cdot 4)$ & 0 & $4(6 \cdot 8)$ & $40(42 \cdot 6)$ \\
\hline \multicolumn{7}{|l|}{ Mild treatment: } \\
\hline Total respondents & 100 & 303 & 789 & 88 & 60 & 92 \\
\hline $\begin{array}{l}\text { No (\%) of respondents accepting } \\
\text { treatment }\end{array}$ & $19(19)$ & $80(26 \cdot 4)$ & $167(21 \cdot 2)$ & $2(2 \cdot 3)$ & $7(11 \cdot 7)$ & $54(58 \cdot 7)$ \\
\hline
\end{tabular}

mation about their diagnosis and details about the treatment. They are told whether the aim of treatment is to cure the cancer or to control it, but seldom are patients given statistics and likelihoods to help them make their decisions. This information is usually given only if the patient asks for it. A hypothetical framework was specifically chosen for this study to avoid demoralising the patients and to prevent them thinking that their answers to the questions would influence their treatment.

All the patients who were given the questionnaire, however, knew that they were about to receive chemotherapy and thus considered the questions seriously. This group thus excluded those patients for whom chemotherapy was an unacceptable treatment option because they would not have been referred to the unit. To this extent the group was not representative of an entire population of patients with cancer. There are no numbers available about the percentage of all patients with cancer who would decline chemotherapy if offered. In clinical practice, however, only a small proportion of patients with cancer refuse chemotherapy, and the numbers are therefore probably close to those that would be obtained in an unselected sample of patients with cancer. The patients' responses were compared with those of a matched control group without cancer. This control group was carefully matched for age and sex, as well as for occupation and hence educational and social class. It was therefore hoped that the attitude of the control group would represent that of the patient group before they had developed cancer. The large differences that emerged between these two groups suggest that patients' attitudes to treatment change dramatically when they are given the diagnosis of cancer. Faced with the reality of the diagnosis, as opposed to the purely theoretical possibility, patients are likely to accept any treatment that offers them some possible benefit and hope, however slight. It suggests that people with cancer find it difficult to accept circumstances in which there are no therapeutic options, and it might be argued that their response represents a feeling of panic in a crisis. Thus when asked about relief of symptoms $43 \%$ of the patients accepted intensive treatment when the chance of relief was very small.

Although doctors may have the facts and statistics available they inevitably make subjective judgments in recommending a particular treatment option to patients. Assessments of patients' quality of life and their levels of anxiety and depression by doctors and nurses correlate poorly with those made by the patients themselves. ' This study illustrates how difficult it is to suggest that doctors and nurses should be, or indeed are, in a position to advise patients what treatment to have based on what they might want in a similar situation. Firstly, different groups of doctors and nurses had significantly different views of what they would accept for themselves. Secondly, there was a wide variability within individual groups. It is interesting to note the large differences between the two groups of cancer specialists, with the medical oncologists having a more optimistic view of the benefit of chemotherapy than the radiotherapists, who represented the most pessimistic group (table II). The radiotherapists' response has to be taken with a degree of caution because of the low rate of reply $(37 \%)$ compared with that of the oncologists $(78 \%)$, $(\mathrm{p}<0.0001)$. The same caveat applies to the responses of the nurses. It is often said that doctors are overenthusiastic about offering patients intensive treatment when the chance of benefit is small, but these data suggest that the patients themselves were considerably more enthusiastic than their doctors.

The patients with cancer appeared to regard a minute chance of possible benefit as worth while, whatever the cost. It would be easy to conclude that this is an irrational decision resulting from the tremendous stress imposed on these patients by their disease. This is by no means clear, however; it may be that the only people who can evaluate such life and death decisions are those faced with them.

The patients' responses in this study suggest that a high degree of toxicity is acceptable, even if only a slight chance of cure is offered. It seems probable that this value cannot be appreciated when a person is well and that attitude changes appreciably when a person is faced with what is perceived as a life threatening disease.

1 Cassileth BR, Zupkis RV, Sutton-Smith K, March BA. Information and participation preferences among cancer patients. Ann Intern Med 1980;92: $832-6$

2 Degner LF, Russell CA. Preferences for treatment control among adults with cancer. Res Nurs Health 1988;11:367-74.

3 Slevin MI. Talking about cancer: how much is too much? Br f Hosp Med 1987; July:56-9.

+ Faden RR, Becker C, Lewis C, Freeman J, Faden AI. Disclosure of information to patients in medical care. Med Care 1981; xix:718-33.

5 McNeil BJ, Woichelshaum R, Pauker SG, Speech and survival ureoffs between quality and quantity of life in laryngeal cancer. $N$ Engl J Med between quality

. lung cancer. $N$ Engl I Med 1978;299:1397-401.

7 Slevin ML, Plant H, Lynch D, Drinkwater J, Gregory WM. Who should Slevin ML, Plant H, Lynch D, Drinkwater J, Gregory WM. Who should
measure the quality of life, the doctor or the patient? Br $\mathcal{J}$ Cancer 1988;57:109-112

(Accepted 9 March 1990

\section{ANY QUESTIONS}

\section{Are there any lasting physical consequences of competitive swimming?}

Provided the person is physically fit there are no lasting consequences of competitive swimming. Most of the problems related to aquatic sports are caused outside competitions, and the only lasting physical consequences are related to competing in an unfit state or before a swimming problem has resolved. This is commonly seen when individuals persist with competitive swimming following such conditions as stress fractures of the pars interarticularis and chronic shoulder injuries such as occur in butterfly swimmers. In water polo, however, like any other contact sport, there may be some residual disability from damaged fingers, etc, when the athlete persists in competing before complete healing has occurred. - J M CAMERON, professor of forensic medicine and honorary medical adviser to the Amateur Swimming Association 